\title{
Partial Sequence Analysis of Cytochrome b Gene by FINS Technique Reveals Fraud Sambar Meat in Wild Food Restaurant
}

\begin{abstract}
Thanat Vorajinda ${ }^{1}$, Chavin Chaisongkram ${ }^{2}$, Wibhu Kutanan ${ }^{3}$, and Khemika Lomthaisong ${ }^{1 *}$
\end{abstract}

${ }^{1}$ Forensic Science Program, Faculty of Science, Khon Kaen University, Khon Kaen 40002, Thailand

${ }^{2} Z$ Zoological Organization Khon Kaen Zoo, 40002, Thailand

${ }^{3}$ Department of Biology, Faculty of Science, Khon Kaen University, Khon Kaen 40002, Thailand

*Corresponding author.E-mail: khemlo@kku.ac.th

https://doi.org/10.12982/CMUJNS.2019.0031

Received: February 26, 2019

Revised: March 27, 2019

Accepted: April 5, 2019

\begin{abstract}
Species identification of animals in the Cervidae by sequence comparison of the Cytochrome b (Cyt b) gene using FINS method is focused in this study. The Cyt b fragments of seven species in the Cervidae, including rusa deer (Cervus timorensis), sambar deer (C. unicolor), sika deer (C. nippon), hog deer (C. porcinus), axis deer (C. axis), Eld's deer (C. eldi) and barking deer (Munticacus muntjak) were amplified by our own designed primers. The amplicons (322 bp) were sequenced and their partial sequences (263 bp) were then analyzed. Barking deer showed the highest value of genetic diversity within species $(\pi=0.0364 ; h=0.9)$. The phylogenetic analysis had shown that the partial sequence of Cyt b gene can be used to classify most studied species in Cervidae accurately, except for sambar and rusa deer that cannot be differentiated. Hence, species identification of unknown meat samples was then performed by this method. Referenced Cyt b sequences of wild boar (Sus scrofa), favorite meat in wild food restaurant, and the Cyt b sequence of known sambar tissue samples were additionally compared. The genetic distances indicated that unknown meat samples were presumably wild boar. Although this method cannot differentiate sambar from rusa deer, this study
\end{abstract}


will be useful for wildlife forensic particularly when screening examination of irrelevant samples and fraud sambar meats identification are necessary.

Keywords: Cytochrome $b$ gene, DNA variation, Cervidae, FINS

\section{INTRODUCTION}

Nowadays, large numbers of endangered wildlife animals listed in the CITES appendices are smuggled for illegal trades. In this case, wildlife forensic science has been used as a significant tool to prosecute the smuggler. Studies on wildlife forensic science have been reviewed (Johnson et al., 2014). Most of them have focused on species identification, for which methods based on DNA analysis are more advantageous than those of traditional morphological identification because they can be applied on different types of samples, for example, processed animal parts, derivatives within Traditional Chinese Medicine (TCMs) and objects made from animal parts (Iyengar, 2014) DNA markers used for species identification must be species-specific. These sequences can be found in both nuclear and mitochondrial DNA. However, mitochondrial DNA is more preferable because it contains high copy numbers and hardly degrades.

Many studies have shown that species-specific sequences of mitochondrial DNA, such as ND1 (Kitpipit et al., 2012; Welton et al., 2013), COI (Wilson-Wilde et al., 2010), $16 S$ rRNA (Imaizumi et al., 2007), Cyt b (Jun et al., 2011), D-loop (Fumagalli et al., 2009; Gupta et al., 2011) and ITS-2 (Clarke et al., 2006) can be used to identify animal species. Molecular techniques like PCR-RFLP have been conducted to discriminate DNA polymorphisms of a target sequence for species identification, for example $C y t b$ for identification of fish species in the Cyprinidae family (Chen et al., 2011) and $16 S$ rRNA for sea cucumber identification (Wen et al., 2010). However, the questioned species may not be revealed when the PCR-RFLP profile of an unknown sample does not match with any of those reference species. Hence, a technique called FINS (Forensically Informative Nucleotide Sequencing) has been introduced. This technique identifies species by using phylogenetic analysis in which DNA sequence of a sample is compared with known DNA sequences in the database (Li et al., 2011)). The FINS technique has been reported as a successful method for determination of authentic seafood ingredients including, octopus (Espineira et al., 2010), jelly fish (Armani et al., 2013) and ling fish (Santaclara et al., 2014). This technique has also been applied for mislabeling investigation of pet canned food (Armani et al., 2015). 
In wildlife forensic science, species identification based on DNA analysis can be performed by PCR amplification of target DNA using species-specific primer, i.e. examination of a shawl woven from Tibetan antelope hair, a protected species listed in the CITES appendix I (Lee et al., 2006) and identification of protected buffalo meat in Sri Lanka (Rajapaksha et al., 2003). Nevertheless, species identification by PCR amplification with species-specific primer cannot differentiate samples of different subspecies, in which case determination of genetic variation by FINS will cope with this limitation as shown by Gupta et al. (2013) who employed the technique to distinguish wild pigs from domestic ones. However, species identification by FINS needs reference DNA data from related species. The lack of reference DNA may cause misinterpretation. Unknown samples of a viverrid were misidentified as coming from hyena due to the insufficient data of Veverricula indica $16 S$ rRNA sequences in the NCBI database (Sahajpal and Goyal, 2010).

In Thailand, large numbers of livestock belong to the Cervidae family, i.e. sika, rusa, hog and sambar deer. The latter is a protected species; however, due to the popularity of its meat, farming sambar deer is allowed albeit only through legal permission. Since its farming practice is subject to tight regulation, the samba deer meat is highly valued and costly. Owing to this, meats of other animals are sometimes deceitfully advertised as the meat of sambar deer, necessitating proper identification of the meats. Although DNA barcoding (COI) has been successfully used to identify sambar deer meat, the accuracy of this method depends on the quality of the database (Kumar et al., 2012). Moreover, for species identification by DNA barcoding, a target size of 5' COI region is approximately 650 bp (Trivedi et al., 2016). This may be troublesome when highly degraded DNA samples are performed. Hence, an alternative method for sambar deer meat identification by comparing $C y t b$ sequences using FINS technique is focused in this study. According to our results, the FINS technique has proved a useful tool for species identification enabling us to forensically trace the origins of the meats sold in wildlife restaurants across Thailand.

\section{MATERIALS AND METHODS}

\section{Samples}

Blood samples of five individuals per species including C. timorensis, C. unicolor, $C$. nippon, $C$. porcinus, $C$. axis, C. eldi and M. muntjak were kindly provided by Khon Kaen Zoo under the permission from the Animal Ethics Committee (ACUC-KKU-31/2559). Ten milliliters of blood was taken into a test tube containing $500 \mu \mathrm{l}$ of $0.5 \mathrm{M}$ EDTA. Blood samples were then kept at 
$4^{\circ} \mathrm{C}$ until DNA extraction was performed. For unknown meats, five samples (100 g each) were taken from wild food restaurants in Thailand (two from Prajeenburi, two from Phra Nakorn Sri Ayuttaya and one from Nakhon Nayok province). The meats were kept at $4{ }^{\circ} \mathrm{C}$ until they were used. For reference, meat (MC) and liver (LC) of C. unicolor and C. timorensis antler (HC) were also provided by Khon Kaen Zoo.

\section{DNA isolation}

DNA extraction from the blood samples was conducted using QIAamp ${ }^{\circledR}$ DNA Blood Mini Kit (QIAGEN, Germany). Briefly, $200 \mu 1$ of each sample was incubated with proteinase $\mathrm{K}$ at $56^{\circ} \mathrm{C}$ for $10 \mathrm{~min}$. Then, ethanol was added and the mixture was transferred onto QIAamp mini spin column. This column was centrifuged to get rid of the liquid. The column was subsequently washed twice. The DNA was then eluted from the column. For meat and antler samples, DNA extraction using NucleoSpin ${ }^{\circledR}$ kit was performed according to the manufacturer's instruction. Twenty-five mg of each tissue sample was incubated with proteinase $\mathrm{K}$ at $56^{\circ} \mathrm{C}$ for $3 \mathrm{~h}$. Then, $100 \%$ ethanol was added and the mixture mixed before being transferred to NucleoSpin ${ }^{\circledR}$ tissue column. The liquid was removed from the column by centrifugation. The column was then washed and DNA was subsequently eluted. The quantity and quality of DNA solution were examined by NanoDrop spectrophotometer.

\section{Primer design}

The nucleotide sequences of $C y t b$ genes from animals in the Cervidae were obtained from GenBank database (http://www.ncbi.nlm.nih.gov/genbank). Cyt $b$ sequences of C. porcinus (DQ379301.1), C. axis (AY607040.1), C. eldi (AY157735.1), C. nippon (D32192.1), C. timorensis (AF423200.1), C. unicolor (AF423201.1) and M. muntijak (AF042718.1) were multiple aligned using ClustalW2 program (http://www.ebi.ac.uk/Tools/msa/clustalw2/). Primers were designed from conserved regions by OligoAnalyzer 3.1 (https://www.idtdna. com/calc/analyzer).

\section{PCR amplification}

The PCR reaction containing $0.2 \mathrm{ng}$ DNA, $0.4 \mu \mathrm{M}$ of each primer and 1xPCR Master Mix (Vivantis, Malysia) was set up. A total volume of $25 \mu 1$ was made by adding deionized water. The amplification was then performed in a Thermal Cycler with the following cycling conditions: initial denaturation step at $94^{\circ} \mathrm{C}$ for $2 \mathrm{~min}$ followed by 35 cycles of $94^{\circ} \mathrm{C} 30 \mathrm{sec}, 57^{\circ} \mathrm{C} 30 \mathrm{sec}$, $72^{\circ} \mathrm{C} 30 \mathrm{sec}$ and a final extension at $72^{\circ} \mathrm{C} 5 \mathrm{~min}$. The PCR product was 
subsequently analyzed on $1.5 \%$ agarose gel. The size of the amplicon was estimated from the 100 bp DNA marker (VIVANTIS, Malaysia).

\section{DNA sequencing and haplotype analysis}

The PCR products were sent to Macrogen (South Korea) for DNA sequencing with our designed primers. The nucleotide sequences of forward and reverse strands from each sample were aligned by BioEdit 7.0 program (Hall, 1999). Fine adjustment was manually performed with visual inspection. These sequences were then assembled. The sequences of all samples were multiple aligned. Fault insertion and deletion of the bases were manually edited. Nucleotide sequences were trimmed at both ends to produce the equal lengths of the sequences. From the result by multiple alignment, the polymorphic sites of nucleotide were examined using DnaSP 5.0 (Librado and Rozas, 2009). Then, nucleotide diversity $(\pi)$ and haplotype diversity $(h)$ were determined.

\section{Phylogenetic analysis}

Thirty-five sequences of the $C y t b$ gene obtained from this study were aligned with seven sequences of species belonging to the Cervidae familyretrieved from the GenBank database, using MEGA version 6 . A fragment of $263 \mathrm{bp} C y t b$ gene was selected for comparison by means of neighbor-joining $(\mathrm{NJ})$ tree. The genetic distances were also computed using K2-parameter model with 2000 bootstrap re-samplings.

\section{Species identification by FINS methods}

The DNA extraction from unknown meat samples, reference samples of C. unicolor (meat, liver) and C. timorensis (antler) were conducted. PCR amplification of the $C y t b$ gene was then performed followed by DNA sequencing of amplicon as described earlier. The DNA sequences were compared with reference sequences of the $C y t b$ gene of known cervids.

\section{RESULTS}

\section{Primer design}

Based on the result from the alignment of $C y t b$ gene sequences from seven species of the Cervidae, primers were designed from conserved regions. Then, nucleotide positioned 182-200 and 481-503 were chosen for forward and reverse primers, respectively (Figure 1). As a result, a DNA fragment with a length of $322 \mathrm{bp}$ was amplified. 
MM 1 ATGACAAATATTCGAAAAACCCACCCACTAATAAAAATTGTAAACAATGCATTCATTGAT 60 CE 1 ATGACCAATATCCGAAAAACCCACCCATTAATAAAAATTGTGAACAACGCATTTATTGAC 60 CN 1 ATGACTAATATCCGAAAAACCCACCCATTAATAAAAATTGTAAACAACGCATTCATTGAC 60 CT 1 ATGACCAATATCCGAAAAACCCACCCATTAATAAAGATTGTAAACAACGCATTCATTGAC 60 CU 1 ATGACCAATATCCGAAAAACCCACCCATTAATAAAGATTGTAAACAACGCATTCATTGAC 60 CP 1 ATGATCAATATCCGAAAAACCCACCCATTAATAAAAATTGTAAACAACGCATTCATTGAC 60 CA 1 ATGATCAACCTCGGAAAAACCTCCCCATTAATAAAAATTGTAAACAACGCATTCATTGAC 60 $* * * * * * * * * * * * * * * * * * * * * * * * * * * * * * * * * * * * * * * * * * * * * *$

MM 61 CTCCCAGCCCCATCAAATATCTCATCTTGATGAAACTTTGGCTCCCTACTAGGAATCTGC 120 CE 61 CTCCCAGCCCCATCAAATATTTCATCCTGATGAAATTTCGGCTCCTTGCTAGGAGTTTGC 120 CN 61 CTCCCCGCCCCATCAAATATTTCATCCTGATGAAATTTCGGCTCCCTACTAGGAATTTGT 120 CT 61 CTCCCAGCCCCATCAAATATTTCATCCTGATGAAATTTCGGCTCCTTACTAGGAATTTGT 120 CU 61 CTCCCAGCCCCATCAAATATTTCATCCTGATGAAATTTCGGCTCCTTACTAGGAATTTGT 120 CP 61 CTCCCAGCACCATCAAATATTTCATCCTGATGGAACTTCGGCTCTCTGCTAGGAGTCTGC 120 CA 61 CTCCCAGCCCCATCAAATATTTCATCCTGATGGAACTTCGGCTCTTTGCTAGGAGTCTGC 120 $* * * * * * * * * * * * * * * * * * * * * * * * * * * * * * * * * * * * * * * * * * * * * * *$

MM 121 TTAATTCTACAAATCCTCACAGGCCTATTTCTAGCAATGCACTACACATCCGACACAATA 180 CE 121 CTAATTCTACAAATCCTCACAGGCCTATTTCTAGCAATACACTACACATCTGATACAATA 180 CN 121 CTAATCCTACAAATCCTTACAGGCCTATTCCTAGCAATACACTATACATCTGACACAATA 180 CT 121 CTAATCCTACAAATCATCACAGGCCTGTTCCTAGCAATACACTATACATCCGATACAATA 180 CU 121 CTAATCCTACAAATCATCACAGGCCTATTCCTAGCAATACACTATACATCCGATACAATA 180 CP 121 TTAATTCTACAAATCCTCACAGGCCTATTCTTGGCAATACACTATACATCCGACACAATA 180 CA 121 TTAATTCTACAAATTCTCACGGGCTTATTCTTAGCAATACACTATACATCTGATACAATA 180 $* * * * * * * * * * * * * * * * * * * * * * * * * * * * * * * * * * * * * * * * * * * * *$

\section{5'-CAGCATTTTCCTCTGTTAC-3'}

MM 181 ACAGCATTCTCCTCGGTCACCCATATCTGCCGAGACGTCAACTATGGCTGAATCATCCGA 240 CE 181 ACAGCATTTTCCTCTGTCACCCATATCTGTCGAGATGTCAACTATGGCTGAATTATTCGA 240 CN 181 ACAGCATTTTCCTCTGTCACCCATATCTGTCGAGATGTCAACTATGGTTGAATTATCCGA 240 CT 181 ACAGCATTTTCCTCTGTTACCCATATCTGCCGAGATGTCAATTATGGCTGAATCATTCGA 240 CU 181 ACAGCATTTTCCTCTGTTACCCATATCTGCCGAGATGTCAATTATGGCTGAATCATTCGA 240 CP 181 ACAGCATTCTCCTCTGTTACCCATATTTGCCGAGACGTCAATTACGGCTGAATTATTCGA 240 CA 181 ACAGCATTCTCCTCTGTCACTCATATCTGTCGAGACGTCAACTACGGCTGAATTATTCGA 240 $* * * * * * * * * * * * * * * * * * * * * * * * * * * * * * * * * * * * * * * * * * * * * * * *$

MM 241 TATATACACGCAAACGGAGCATCAATATTTTTCATTTGCTTATTTATTCATGTAGGACGA 300 CE 241 TACATACACGCAAACGGAGCATCAATATTTTTCATCTGTCTATTCATACATGTAGGACGA 300 CN 241 TACATACACGCAAACGGGGCATCAATATTTTTCATCTGCCTATTCATACATGTAGGACGA 300 CT 241 TATATACACGCAAACGGGGCATCAATATTTTTCATCTGTCTATTTATACACGTAGGACGA 300 CU 241 TATATACACGCAAACGGGGCATCAATATTTTTCATCTGTCTATTTATACACGTAGGACGA 300 CP 241 TATATGCACGCAAATGGAGCATCAATATTTTTTATCTGCCTATTTATACATGTAGGACGA 300 CA 241 TATATGCACGCAAATGGAGCATCAATATTTTTTATTTGCTTATTTTTACATGTAGGACGA 300 $* * * * * * * * * * * * * * * * * * * * * * * * * * * * * * * * * * * * * * * * * * * * * * * *$

MM 301 GGCCTATACTATGGATCATACACCTTCCTAGAAACATGAAACATTGGAGTGATTCTTTTA 360 CE 301 GGCCTGTACTACGGATCATATACCTTTCTAGAGACATGAAACATTGGAGTAATTCTCCTA 360 CN 301 GGCCTGTACTACGGATCATATACTTTTCTAGAGACATGAAACATCGGAGTAATTCTCCTA 360 CT 301 GGCCTGTACTACGGATCATACACTTTTCTAGAGACATGAAACATCGGAGTAATCCTCCTA 360 CU 301 GGCCTGTACTACGGATCATACACCTTTCTAGAGACATGAAACATCGGAGTAATCCTCCTA 360 CP 301 GGCCTGTACTACGGATCATACACCTTTCTAGAAACATGAAACATTGGAGTAATCCTCCTA 360 CA 301 GGTCTGTATTACGGATCATATACCTTTTTAGAAACATGAAACATTGGAGTAATCCTCCTA 360 $* * * * * * * * * * * * * * * * * * * * * * * * * * * * * * * * * * * * * * * * * * * * * * *$ 
MM 361 TTTACAGTTATAGCCACGGCATTCGTAGGATATGTTTTACCATGAGGACAAATATCATTT 420

CE 361 TTTACAGTTATAGCAACAGCATTCGTAGGGTATGTCTTACCATGAGGACAGATATCATTC 420

CN 361 TTTACAGTTATAGCCACAGCATTCGTAGGATATGTCCTACCATGAGGACAAATATCATTC 420

CT 361 TTTACAGTTATAGCCACAGCATTCGTAGGGTATGTCCTACCATGAGGACAAATATCATTC 420

CU 361 TTTACAGTTATAGCCACAGCATTCGTAGGGTATGTTCTACCATGAGGACAAATATCATTC 420

CP 361 TTCACGGTTATAGCCACAGCATTCGTAGGATATGTCCTACCATGAGGACAAATATCATTC 420

CA 361 TTTACAGTTATAGCCACAGCATTTGTGGGATACGTCCTACCATGAGGACAGATATCATTC 420

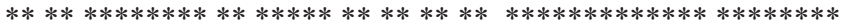

MM 421 TGAGGAGCAACAGTCATCACTAACCTCCTTTCAGCAATTCCATATATTGGCACAAACTTA 480

CE 421 TGAGGAGCAACAGTCATCACCAACCTCCTCTCAGCAATTCCATACATCGGCACAAATCTA 480

CN 421 TGAGGAGCAACAGTCATTACCAACCTCCTCTCAGCAATTCCATATATTGGCACAAACCTA 480

CT 421 TGAGGAGCAACAGTCATTACCAAACTTCTCTCAGCAATTCCATATATTGGTACAAACCTA 480

CU 421 TGAGGAGCAACAGTCATTAACAACCTTCTCTCAGCAATTCCATATATTGGTACAAACCTA 480

CP 421 TGAGGGGCAACAGTTATTACCAACCTCCTCTCAGCAATCCCTTACATCGGCACAAATCTA 480

CA 421 TGAGGAGCAACAGTTATTACCAATCTCCTCTCAGCAATCCCTTACATTGGTACAAATCTA 480 $* * * * * * * * * * * * * * * * * * * * * * * * * * * * * * * * * * * * * * * * * * * * *$

\section{3'-CAGCTTACTTAGACTCCTCCGAA-5'}

MM 481 GTCGAATGAATCTGAGGAGGCTTTTCAGTTGATAAAGCAACCCTCACCCGATTCTTTGCC 540

CE 481 GTCGAATGAATCTGAGGGGGCTTTTCAGTAGATAAAGCAACCCTGACCCGATTTTTCGCT 540

CN 481 GTCGAATGGATCTGAGGGGGCTTCTCAGTAGATAAAGCAACCCTAACCCGATTTTTCGCT 540

CT 481 GTCGAATGAATCTGAGGAGGCTTTTCAGTAGATAAAGCCACCCTAACCCGATTCTTTGCT 540

CU 481 GTCGAATGAATCTGAGGAGGCTTTTCAGTAGATAAAGCCACCCTAACCCGATTTTTTGCT 540

CP 481 GTCGAATGAATCTGAGGGGGCTTTTCAGTAGACAAAGCAACCTTAACCCGATTCTTCGCT 540

CA 481 GTCGAATGAATTTGAGGAGGCTTTTCAGTAGATAAAGCAACCCTAACCCGATTTTTCGCT 540 $* * * * * * * * * * * * * * * * * * * * * * * * * * * * * * * * * * * * * * * * * * * * * * * *$

Figure 1. Multiple sequence alignment of the $C y t b$ gene from 7 species of the Cervidae including M. muntijak (MM), C. eldi (CE), C. nippon (CN), C. timorensis (CT), C. unicolor (CU), C. porcinus (CP) and C. axis (CA). Forward and complementary sequences of reverse primers are shown in bold texts. Asterisk $(*)$ represents identical nucleotides.

\section{PCR results}

DNAs extracted from the animal blood and meat samples were quantified and diluted to $50 \mathrm{ng} / \mathrm{ul}$ for PCR amplification. Optimal melting temperature for PCR was also investigated. As expected from the primer design result, the amplicon was 322 bp long in all samples (Figure 2), indicating successful primer design. 


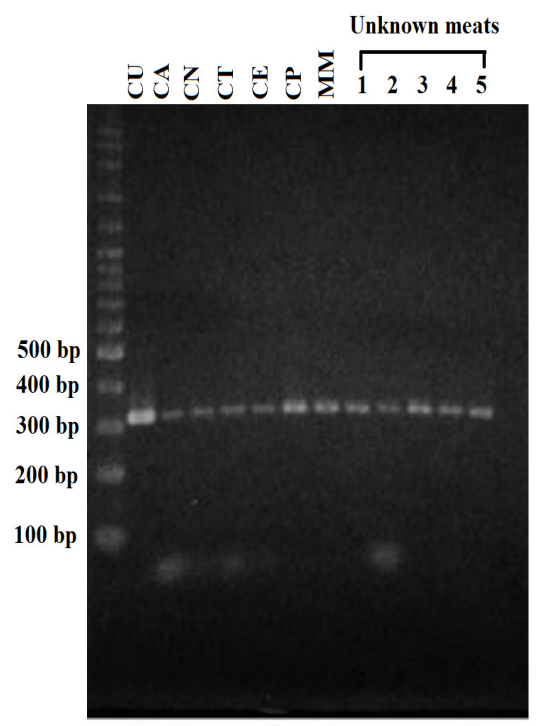

(A)

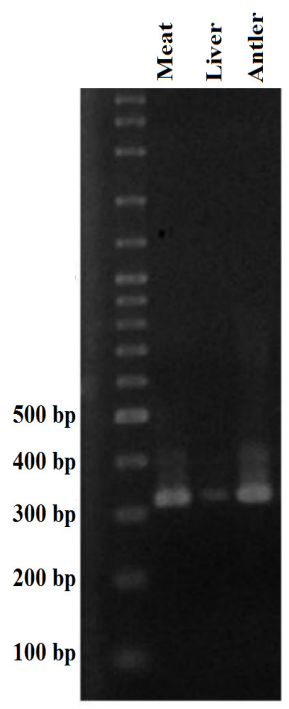

(B)

Figure 2. PCR amplification of the Cyt $b$ gene on DNA extracted from animal blood samples of 7 cervids and from unknown meat samples (A) meat, liver of $C$. unicolor and antler of $C$. timorensis (B) using the designed primer.The size of the amplicon is compared with $100 \mathrm{bp}$ DNA ladder (M). N represents negative control.

\section{Haplotype diversity}

The $263 \mathrm{bp}$ nucleotide sequences of $322 \mathrm{bp}$ PCR products from each species were analyzed for haplotype and nucleotide diversity. Unexpectedly, the $C y t$ bene from C. unicolor, C. timorensis, C. nippon and C. axis samples had no sequence variation as indicated by one haplotype in each species (Table 1). On the contrary, the DNA sequences of the Cyt $b$ gene from M. muntjak samples showed highest variation $(h=0.9 ; \pi=0.03640)$ in which four haplotypes were identified. For $C$. eldi and $C$. porcinus, two haplotypes were found. 
Table 1. Number of haplotypes, haplotype diversity and nucleotide diversity in the $C y t b$ gene (263 bp) of the cervids.

\begin{tabular}{lcccc}
\hline Species & Sample no. & $\begin{array}{c}\text { Number of } \\
\text { haplotype }\end{array}$ & $\begin{array}{c}\text { Haplotype } \\
\text { diversity (h) }\end{array}$ & $\begin{array}{c}\text { Nucleotide } \\
\text { diversity }(\boldsymbol{\pi})\end{array}$ \\
\hline C. unicolor & $1-5$ & 1 & 0 & 0 \\
C. axis & $6-10$ & 1 & 0 & 0 \\
C. nippon & $11-15$ & 1 & 0 & 0 \\
C. timorensis & $16-20$ & 1 & 0 & 0 \\
C. eldi & $21-25$ & 2 & 0.5 & 0.00190 \\
A. porcinus & $26-30$ & 2 & 0.4 & 0.00152 \\
M. muntjak & $31-35$ & 4 & 0.9 & 0.3640 \\
\hline
\end{tabular}

\section{Phylogenetic analysis}

The 263 bp nucleotide sequences of the $C y t b$ gene from 35 samples were compared and analyzed whether or not they could be used for species identification. In order to prove this, $C y t b$ reference sequences of the cervids retrieved from the GenBank database were also included in the NJ tree (Figure 3). Noteworthy, samples of 5 out of 7 species were classified in the same group as their reference species indicating that the sequence of this $C y t b$ region could be used as DNA target for species identification. Although, the two species of $C$. unicolor and C. timorensis could not be differentiated, most species in the Cervidae could be identified. Moreover, sample no.12 was grouped as $C$. axis instead of $C$. nippon. Although, this sample was subjected to repeated analyses, the same result was achieved indicating the possibility of mislabeling. 


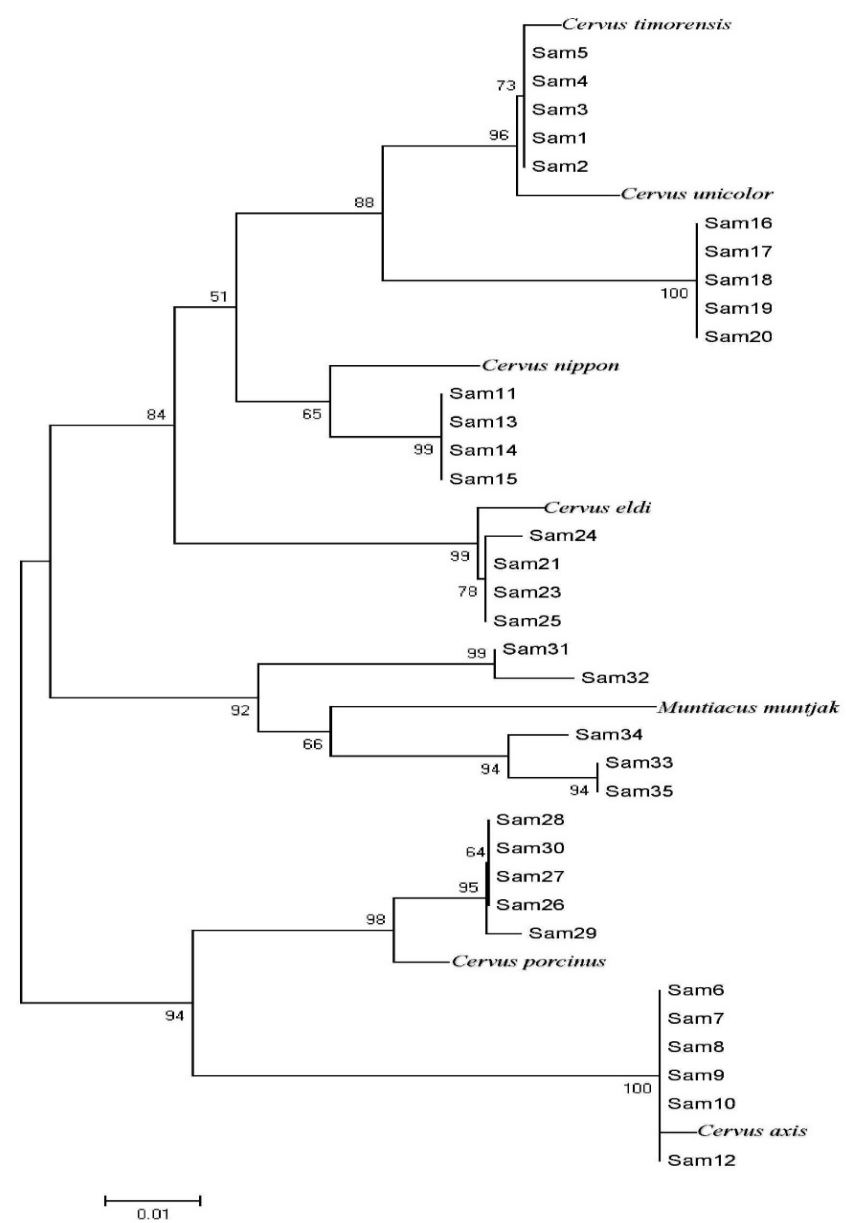

Figure 3. The neighbor joining (NJ) tree of the $C y t b$ gene from the cervid samples compared with those of reference sequences from the database. Bootstrap values were indicated on each node.

\section{Species identification}

For FINS technique, the DNA sequences (263 bp) from unknown meats were compared with the reference sequences of $C y t b$ from seven species of the Cervidae and known C. unicolor samples. Additionally, wild boar (S. Scrofa), a popular meat ingredient in wild food restaurants was also included to represent a fraud sambar meat occurrence. Phylogenetic analysis revealed that unknown meats (Unk1-Unk5) were clustered in the same group as wild boar 
(Figure 4). The pairwise distances are shown in Table 2. The variable nucleotide positions of $C y t b$ sequences from sambar deer and wild boar are shown in Table 3.

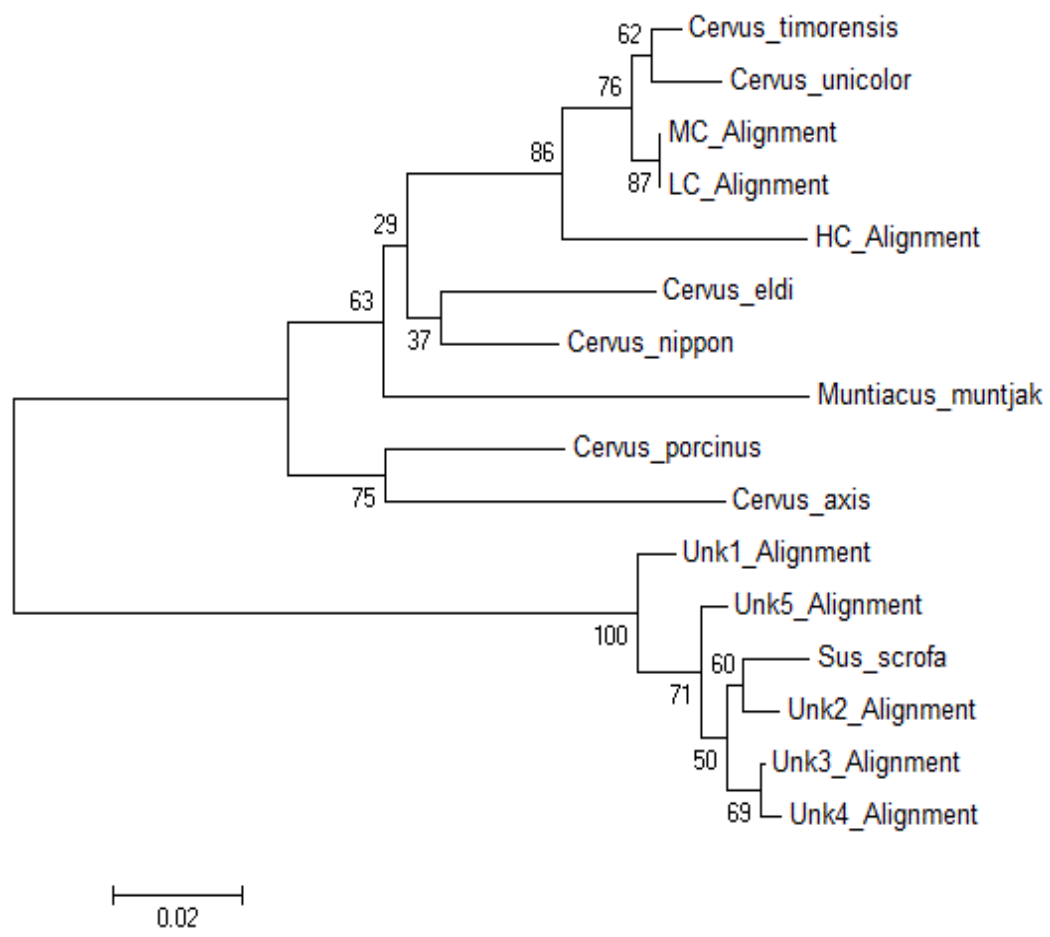

Figure 4. The neighbor joining (NJ) showing the relationships among unknown meats (Unk1-Unk5), wild boar (S. Scrofa), reference cervid species with known $C$. unicolor samples; meats (MC), liver (LC) and $C$. timorensis antlers (HC) based on the alignment of $C y t b$ gene sequences. 


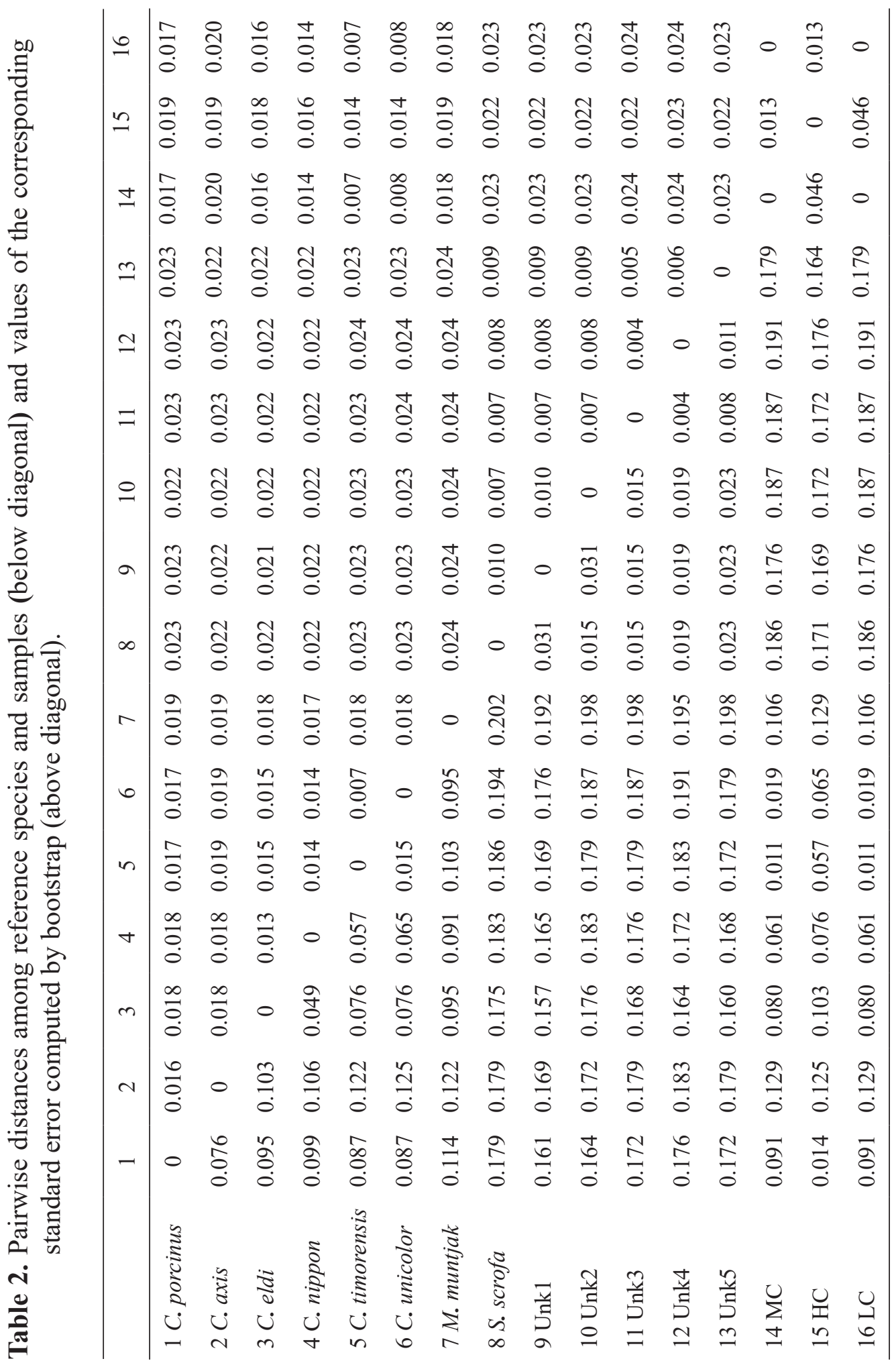




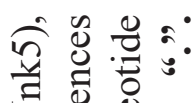

它莺远

灵岕声

已 :

艁

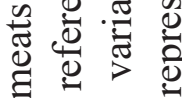

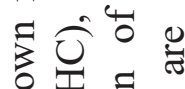

凷.

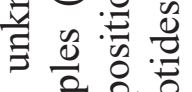

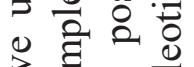

踏

हु

氜

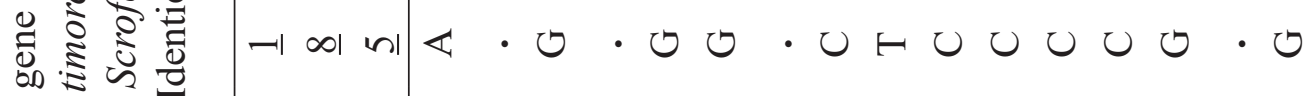

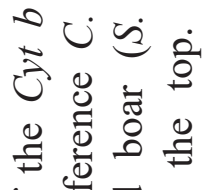

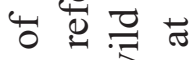

$\tilde{0} \hat{0} \tilde{\theta}$

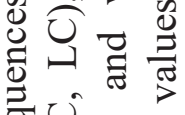

总记蒡

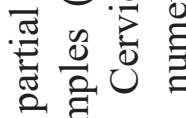

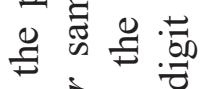

$\exists \frac{\grave{0}}{0} . \Xi$

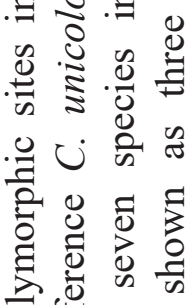

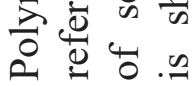

$\dot{m}$

$\frac{0}{\frac{0}{\pi}}$

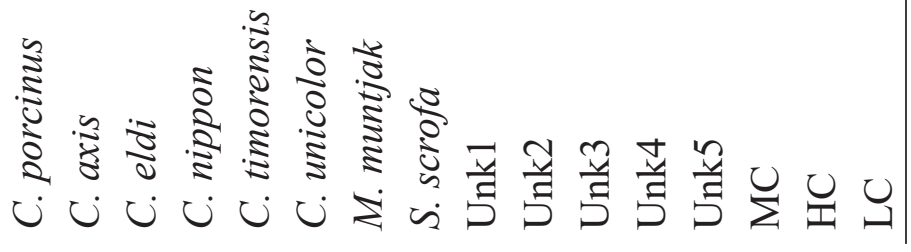




\section{DISCUSSION}

Species identification of sambar deer (C. Unicolor), a member of the Cervidae which is listed in Thailand Wildlife Protection, by FINS technique had been examined in this study. A species-specific sequence of $C y t b$ gene was focused because it has been reported as the locus most frequently used for species identification in forensic science (Parson et al., 2000; Hsieh et al., 2001). Hence, a pair of primers were designed from the $C y t b$ sequences of seven species of the Cervidae. PCR amplification of $C y t b$ by these primers was performed on 35 DNA samples of the cervids ( 5 samples/species). These primers were appropriate to this study as an amplicon of $C y t b(322 \mathrm{bp}$ ) was produced in all samples (Figure 1). The sequence analysis of these amplicons was then conducted. Interestingly, the $C y t b$ sequences of $M$. muntjak showed highest DNA variations, followed by C. eldi and C. porcinus, respectively (Table 1). However, no DNA variations were observed among sequences of the rest of the species. Low genetic variation in this case may be explained by the inbreeding because they have been raised in an enclosed zoo. Studies on species identification by $C y t b$ sequence has been reported earlier but the larger amplified DNA fragments of $358 \mathrm{bp}$ (Parson et al., 2000), 402 (Hsieh et al., 2003) and 421 (Gupta et al., 2013) were investigated.

The NJ tree was then constructed to analyze whether these $C y t b$ sequences could determine the species in the Cervidae (Figure 2). The partial sequences of $C y t$ b (263 bp) were compared with those of reference cervids. Noticeably,samples were classified in the same group as their source species except for samples from $C$. unicolor and $C$. timorensis that cannot be differentiated. These results indicated that the $C y t b$ sequences ( $263 \mathrm{bp}$ ) could be used as a marker for most species identification in the Cervidae. Hence, unknown meats from wild food restaurants were determined for their species using FINS analysis on these sequences (Figure 3). Surprisingly, we found that all unknown meats were classified in the same group as wild boar. To confirm the results that the unknown meats were not belong to Cervidae, controlled samples of C. unicolor samples were also investigated and they were clustered in the same group as $C$. unicolor and C. timorensis. Three forensically informative nucleotide variations were observed between unknown meats and S. scrofa (Table 3 ). In addition, BLAST analysis was also performed in order to ensure that our 
investigations were correct. We found that the sequences of unknown meats (Unk1-5) were close to wild boar with similarity at 94\%, 96\%, 96\%, 96\% and $96 \%$, respectively. These evidences clearly confirm that the analysis of $C y t b$ (263 bp) by the FINS method can identify the source species of meat.

This study has shown that comparison of $C y t b$ partial sequence (263 bp) by the FINS technique is useful for species identification not only for cervids but also other species (if reference sequences are available). Even though, the differentiation between $C$. unicolor and $C$. timorensis cannot be made, this method still has the advantage for wildlife forensic in which it can be used to exclude the irrelevant evidence from consideration. Moreover, as the shorter targeted $C y t b$ had been investigated in this study, DNA analysis for species identification on degraded samples could be achieved (Lopez-Oceja et al., 2016).

\section{CONCLUSION}

Comparison of Cyt $b$ partial sequence (263 bp) by FINS method can be used for species identification of animals in the Cervidae. However, C. unicolor and $C$. timorensis cannot be discriminated by this method. For wildlife forensic application, this study may be useful for preliminary examination to exclude irrelevant evidence.

\section{REFERENCES}

Armani, A., Tinacci, L., Giusti, A., Castigliego, L., Gianfaldoni, D., and Guidi, A. 2013. What is inside the jar? Forensically Informative Nucleotide Sequencing (FINS) of a short mitochondrial COI gene fragment reveals a high percentage of mislabeling in jellyfish food products. Food Research International. 54(2): 1383-1393. https://doi.org/ 10.1016/j.foodres.2013.10.003

Armani, A., Tinacci, L., Xiong, X., Castigliego, L., Gianfaldoni, D., and Guidi, A. 2015. Fish species identification in canned pet food by BLAST and Forensically Informative Nucleotide Sequencing (FINS) analysis of short fragments of the mitochondrial $16 \mathrm{~S}$ ribosomal RNA gene (16S rRNA). Food Control. 50: 821-830. https://doi.org/10.1016/j.food cont.2014.10.018 
Clarke, S.C., Magnussen, J.E., Abercrombie, D.L., McAllister, M.K., and Shivji, M.S. 2006. Identification of shark species composition and proportion in the Hong Kong shark fin market based on molecular genetics and trade records. Conservation Biology. 20(1): 201-211.

Chen, C.H., Hsieh, C.H., and Hwang, D.F. 2012. Species identification of Cyprinidae fish in Taiwan by FINS and PCR-RFLP analysis. Food Control. 28(2): 240-245. https://doi.org/10.1016/j.foodcont.2012.05.012

Espineira, M., Vieites, J.M., and Santaclara, F. 2010. Species authentication of octopus, cuttlefish, bobtail and bottle squids (families Octopodidae, Sepiidae and Sepiolidae) by FINS methodology in seafoods. Food Chemistry. 121(2): 527-532. https://doi.org/10.1016/j.foodchem.2009. 12.042

Fumagalli, L., Cabrita, C.J., and Castella, V. 2009. Simultaneous identification of multiple mammalian species from mixed forensic samples based on mtDNA control region length polymorphism. Forensic Science International: Genetics Supplement Series. 2(1): 302-303. https://doi.org/ 10.1016/j.fsigss.2009.08.009

Gupta, S.K., Thangaraj, K., and Singh, L. 2011. Identification of the source of ivory idol by DNA analysis. Journal of Forensic Sciences. 56(5): 1343-1345. https://doi.org/10.1111/j.1556-4029.2011.01750.x

Gupta, S.K., Kumar, A., Hussain, S.A., and Singh, L. 2013. Cytochrome $b$ based genetic differentiation of Indian wild pig (Sus scrofa cristatus) and domestic pig (Sus scrofa domestica) and its use in wildlife forensics. Science and Justice. 53(2): 220-222. https://doi.org/10.1016/j.scijus. 2012.09.005

Hall, T.A. 1999. BiEdit: A user-friendly biological sequence alignment editor and analysis program for Windows 95/95/NT. Nucleic Acids Symposium Series. 41: 95-98.

Hsieh, H.M., Chiang, H.I., Tsai, L.C., Lai, S.Y., Huang, N.E., Linacre, A., and Lee, J.C.I. 2001. Cytochrome $b$ gene for species identification of the conservative animals. Forensic Science International. 122(1): 7-18. https://doi.org/10.1016/S0379-0738(01)00403-0

Hsieh, H.M., Huang, L.H., Tsai, L.C., Kuo, Y.C., Meng, H.H., Linacre, A., and Lee, J.C.I. 2003. Species identification of rhinoceros horns using cytochrome b gene. Forensic Science International. 136(1-3): 1-11. https://doi.org/10.1016/S0379-0738(03)00251-2

Imaizumi, K., Akutsu, T., Miyasaka, S., and Yoshino, M. 2007. Development of species identification tests targeting the $16 \mathrm{~S}$ ribosomal RNA coding region in mitochondrial DNA. International Journal of Legal Medicine. 212: 184-191. https://doi.org/10.1007/s00414-006-0127-5 
Iyengar, A. 2014. Forensic DNA analysis for animal protection and biodiversity conservation: A review. Journal of Nature Conservation. 22(3): 195-205. http://dx.doi.org/10.1016/j.jnc.2013.12.001

Johnson, R., Wilson-Wilde, L., and Linacre, A. 2014. Current and future directions of DNA in wildlife forensic science. Forensic Science International: Genetics. 10: 1-11. https://doi.org/10.1016/j.fsigen.2013. 12.007

Jun, J., Han, S.H., Jeong, T.J., Park, H.C., Lee, B., and Kwak, M. 2011. Wildlife forensic using mitochondrial DNA sequences: Species identification based on hairs collected in the field and confiscated tanned Felidae leathers. Genes \& Genomics. 33: 721-726. https://doi.org/ 10.1007/s13258-011-0080-7

Kitpipit, T., Tobe, S.S., Kitchener, A.C., Gill, P., and Linacre, A. 2012. The development and validation of a single SNaPshot multiplex for tiger species and subspecies identification-Implication for forensic purposes. Forensic Science International: Genetics. 6: 250-257. https://doi.org/ 10.1016/j.fsigen.2011.06.001

Kumar, U.S., Ratheesh, R.V., Thomas, G., and George, S. 2012. Use of DNA barcoding in wildlife forensics: a study of sambar deer (Rusa unicolor). Forest Science and Technology. 8: 224-226. https:// doi.org/10.1080/21580103.2012.750802

Lee, J.C., Tsai, L.C., Yang, C.Y., Liu, C.L., Huang, L.H., Linacre, A., and Hsieh, H.M. 2006. DNA profiling of Shahtoosh. Electrophoresis. 27: 3359-3362. https://doi.org/10.1002/elps.200600062

Li, M., Zhang, K.Y.B., But, P.P.H., and Shaw, P.C. 2011. Forensically informative nucleotide sequencing (FINS) for the authentication of Chinese medicinal materials. Chinese Medicine. 6: 42-47. https:// doi.org/10.1186/1749-8546-6-42

Librado, P., and Rozas, J. 2009. DnaSP v5: a software for comprehensive analysis of DNA polymorphism data. Bioinformatics. 25(11): 14511452. https://doi.org/10.1093/bioinformatics/btp187

Lopez-Oceja, A., Gamarra, D., Borragan, S., Jimenez-Moreno, S., and de Pancorbo, M.M. 2016. New cyt $b$ gene universal primer set for forensic analysis. Forensic Science International: Genetics. 23: 159-165. https://doi.org/10.1016/j.fsigen.2016.05.001

Parson, W., Pegoraro, K., Niederstatter, H., Foger, M., and Steinlechner, M. 2000. Species identification by means of the cytochrome $b$ gene. International Journal of Legal Medicine.114(1-2): 23-28. 
Rajapaksha, W., Thilakaratne, I., Chandrasiri, A., and Niroshan, T. 2003. Development of PCR assay for identification of buffalo meat. Asian-Australasian Journal of Animal Sciences. 16: 1046-1048. https://doi.org/10.5713/ajas.2003.1046

Sahajpal, V., and Goyal, S.P. 2010. Identification of a forensic case using microscopy and forensically informative nucleotide sequencing (FINS): a case study of small Indian civet (Viverricula indica). Science and Justice. 50: 94-97. https://doi.org/10.1016/j.scijus.2009.07.002

Santaclara, F.J., Perez-Martin, R.I., and Sotelo, C.G. 2014. Developed of a method for the genetic identification of ling species (Genypterus spp.) in seafood products by FINS methodology. Food Chemistry. 143: 22-26. https://doi.org/10.1016/j.foodchem.2013.06.004

Trivedi, S., Abdulhadi, A.A., Ansari, A.A., and Ghosh, S.K. 2016. Role of DNA barcoding in marine biodiversity assessment and conservation: an update. Saudi Journal of Biological Sciences. 23: 161-171. https:// doi.org/10.1016/j.sjbs.2015.01.001

Welton, L.J., Siler, C.D., Linkem, C.W., Diesmos, A.C., Diesmos, M.L., Sy, E., and Brown, R.M. 2013. Dragons in our midst: phyloforensics of illegally traded Southeast Asain monitor lizards. Biological Conservation. 159: 7-15. https://doi.org/10.1016/j.biocon.2012.10.013

Wen, J., Hu, C., Zhang, L., Luo, P., Zhao, Z., Fan, S., and Zu, T. 2010. The application of PCR-RFLP and FINS for species identification used in sea cucumbers (Aspidochirotida: Stichopodidae) products from the market. Food Control. 21: 403-407. https://doi.org/10.1016/j.foodcont. 2009.06.014

Wilson-Wilde, L., Norman, J., Roberson, J., Sarre, S., and Georges, A. 2010. Current issue on species identification for forensic science and the validity of using cytochrome oxidase I (COI) gene. Forensic Science Medicine and Pathology. 6: 233-241. https://doi.org/10.1007/s12024010-9172-y 